\title{
Gene Amplification or Deletion Detection
}

National Cancer Institute

\section{Source}

National Cancer Institute. Gene Amplification or Deletion Detection. NCI Thesaurus.

Code C19133.

Typically, the use of hybridization techniques to detect amplification or deletion of specific DNA sequences. The hybridization signal of a specific sequence is compared to that of a reference sequence, usually one known to be present once per haploid genome. 